\title{
Atomic-resolution STEM-EDS mapping of grain boundary solute segregation in yttria-stabilized zirconia
}

\author{
Bin Feng ${ }^{1}$, Akihito Kumamoto ${ }^{1}$, Nathan R. Lugg ${ }^{1}$, Naoya Shibata ${ }^{1,2}$ and Yuichi Ikuhara ${ }^{1,3,4}$ \\ 1. Institution of Engineering Innovation, The University of Tokyo, Tokyo, 113-8656 Japan. \\ 2. PRESTO, Japan Science and Technology Agency, Saitama, 332-0012, Japan. \\ 3. Nanostructures Research Laboratory, Japan Fine Ceramic Center, Nagoya, 456-8587, Japan. \\ 4. WPI-AIMR Research Center, Tohoku University, Sendai, 980-8577, Japan.
}

Yttria-stabilized zirconia (YSZ) is one of the primary choices for the electrolyte material in solid oxide fuel cells (SOFC), due to its excellent ionic conductivity at high temperatures. Nevertheless, such performance is usually limited by the ionic conductivity at grain boundaries, which is at least two orders of magnitude lower than that of bulk [1]. As a result, many studies have been performed to investigate grain boundary properties in YSZ, focusing on atomic scale structures and chemical inhomogeneity [2, 3]. Despite the fact that the $Y$ segregation in the grain boundaries has been demonstrated [2], the atomicscale mechanism of how solute segregation occurs is still unclear. Probing the grain boundary at atomic scale is therefore critical for a basic understanding of the chemistry at such interfaces, which in turn will provide new strategies for the design of high performance materials. In this study, the local Y distributions near YSZ grain boundaries were directly determined by atomic-resolution elemental mapping using STEM-EDS, equipped with a high-sensitivity silicon drift detector (SDD).

Five types of YSZ $\left(10 \mathrm{~mol} \% \mathrm{Y}_{2} \mathrm{O}_{3}\right.$ doped $\left.\mathrm{ZrO}_{2}\right)$ bicrystals containing different coincident site lattice grain boundaries were first fabricated by diffusion bonding of two single crystals at $1600{ }^{\circ} \mathrm{C}$ for $15 \mathrm{~h}$ [2]. Elemental mapping was performed using a JEOL JEM-ARM200F cold FEG. The accelerating voltage was $200 \mathrm{kV}$, with the convergence semi-angle of $35 \mathrm{mrad}$. The probe size is about $1.0 \AA$ and the beam current is about $130 \mathrm{pA}$. The STEM-EDS system is equipped with double SDD-EDS detectors and the solid angle for the whole collection system is about $1.7 \mathrm{sr}$.

Figure 1a shows a high angular annular dark-field (HAADF) STEM image of a pristine YSZ crystal from the [110] direction. Since the atomic column intensity in HAADF STEM image is approximately proportional to $\mathrm{Z}^{2}$ (where $\mathrm{Z}$ is the atomic number), the bright contrast represent the cation columns intermixed by $\mathrm{Zr}$ or $\mathrm{Y}$. However, it is impossible to discriminate the $\mathrm{Y}$ atoms from the $\mathrm{Zr}$ atoms in HAADF STEM image because the atomic number of $Y(Z=39)$ is very close to $Z r(Z=40)$. Therefore, STEM-EDS mapping - which is able to isolate individual elements based on their X-ray emission lines was carried out to the pristine YSZ. The corresponding elemental maps are shown in Figures $1 \mathrm{~b}(\mathrm{Zr})$ and c (Y). Elemental maps were extracted by selecting the following EDS energies for each element: $\mathrm{Zr}$, using $\mathrm{K}_{\alpha}$ of $15.776 \mathrm{keV}$ and $\mathrm{K}_{\beta}$ of $17.668 \mathrm{keV}$ and $\mathrm{Y}$, using $\mathrm{K}_{\alpha}$ of $14.958 \mathrm{keV}$ and $\mathrm{K}_{\beta}$ of $16.738 \mathrm{keV}$. Such approaches enable us to clearly see the $\mathrm{Y}$ distribution at atomic resolution; the $\mathrm{Y}$ atoms are homogeneously distributed inside the perfect crystal.

Figure 2 shows an example of HAADF STEM image of a $\Sigma 9$ grain boundary indicated by the arrows. This HAADF-STEM image (in addition to others, not shown here) confirms that all the grain boundaries were bonded well at the atomic level without any amorphous or secondary phases. From elemental maps obtained by STEM EDS, we demonstrate that $\mathrm{Y}$ atoms indeed segregate to the grain boundaries. 
Moreover, there are some specific atomic sites which exhibit a stronger $\mathrm{Y}$ signal, suggesting that preferential segregation sites exist. Such segregation behavior can vary drastically depending on the grain boundary characters. In this presentation, we will combine these experimental results with theoretical calculations and discuss the atomic-scale Y segregation mechanisms in detail.

\section{References}

[1] X. Guo and R. Waser, Progress in Materials Science. 51 (2006) p. 151.

[2] N. Shibata et al, Philosophical Magazine. 84 (2004), p. 2381.

[3] M. Yoshiya and T. Oyama, Journal of Materials Science. 46 (2011), p. 4176.

[4] B.F. and N.L. were supported as Japan Society for the Promotion of Science (JSPS) research fellow.
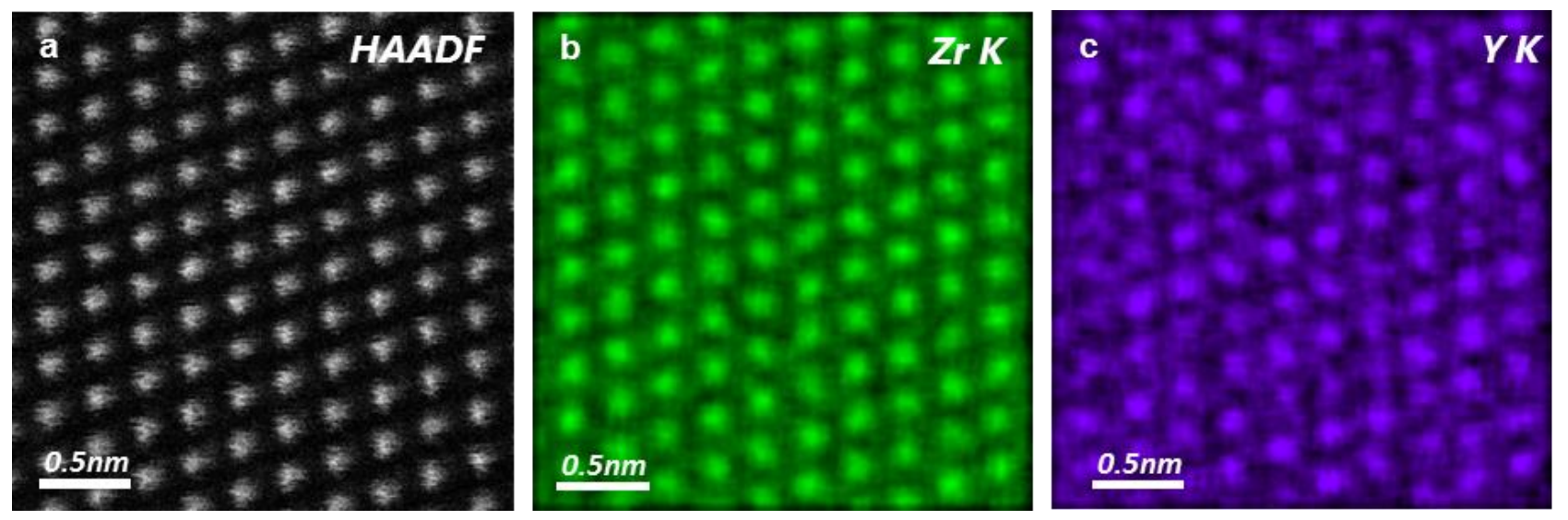

Figure 1. (a) HAADF STEM image of the perfect crystal of YSZ observed from the [110] direction. (b) the corresponding EDS map of Zr K and (c) the EDS map of Y K.

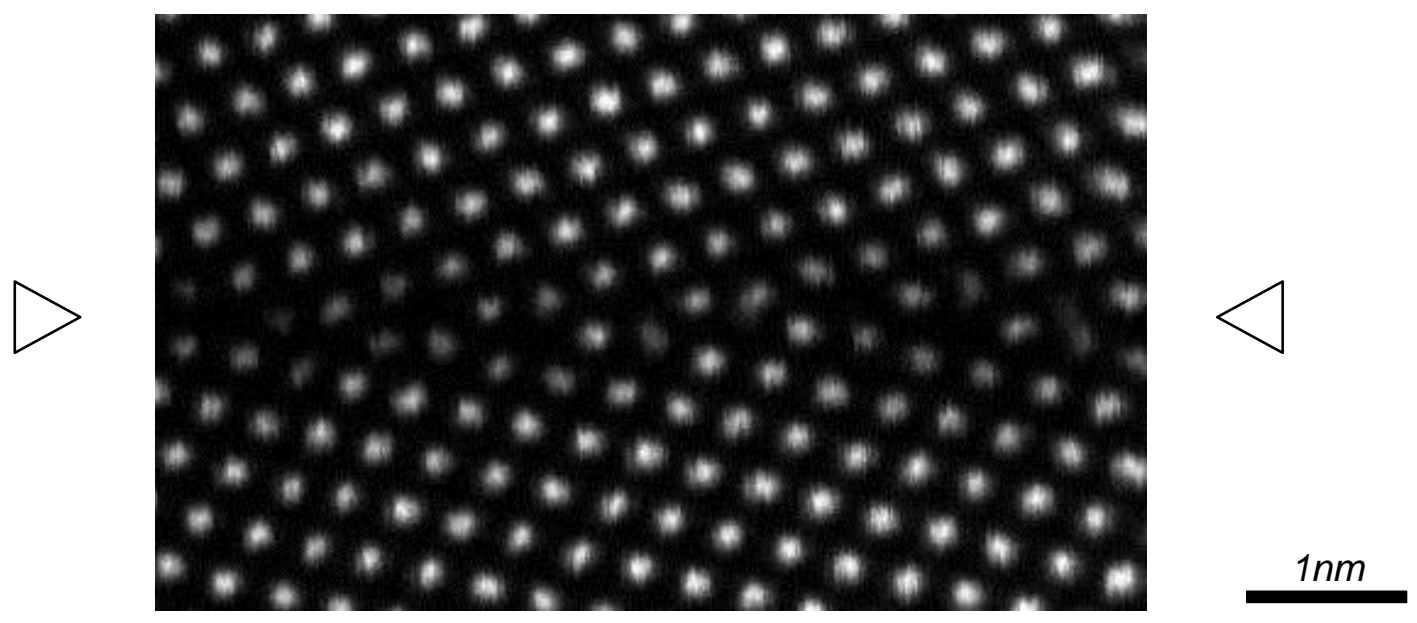

Figure 2. HAADF STEM image of a $\Sigma 9$ model grain boundary. 